\title{
NUMERICAL MODELING OF UNCERTAINTY IN ACOUSTIC PROPAGATION VIA GENERALIZED POLYNOMIAL CHAOS
}

\author{
KHALIL DAMmaK \\ University of Sfax, National School of Engineers of Sfax, Mechanical Modeling and Manufacturing Laboratory (LA2MP), \\ Sfax, Tunisia, and \\ University of Rouen Normandie, LMN, National Institute of Applied Sciences (INSA Rouen), Rouen, France \\ e-mail:khalil.dammak@insa-rouen.fr \\ SAna KoubaA \\ University of Sfax, National School of Engineers of Sfax, Mechanical Modeling and Manufacturing Laboratory (LA2MP), \\ Sfax, Tunisia \\ Abdelkhalak Elhami \\ University of Rouen Normandie, LMN, National Institute of Applied Sciences (INSA Rouen), Rouen, France \\ LASSAAD WALHA, MOHAMED HADDAR \\ University of Sfax, National School of Engineers of Sfax, Mechanical Modeling and Manufacturing Laboratory (LA2MP), \\ Sfax, Tunisia
}

\begin{abstract}
This work aims at increasing the performance prediction for acoustic propagation systems that will operate in the presence of the inevitable parameters uncertainty. In the present contribution, the finite element method is applied to solve an acoustic problem described by the Helmholz equation when the geometric and material properties present uncertainty. The influence of the uncertainty of physical parameters on the pressure field is discussed. The results using the polynomial chaos expansion method are compared with Monte Carlo simulations. It is show that uncertainty levels in the input data could result in large variability in the calculated pressure field in the domain.
\end{abstract}

Keywords: finite element simulation, uncertainty quantification, acoustic propagation, generalized Polynomial Chaos

\section{Introduction}

The guided acoustic propagation is an important field of acoustics. This importance arises as a consequence of the need in reducing or increasing the sound intensity in some regions of wave-guides. The acoustic propagation in cylindrical wave-guides is a common field in structures like automotive and aircraft engines. The Helmholtz equation is normally used to model the propagation of acoustic waves (Cheung and Jin, 1991; Nark et al., 2003, 2005; Taktak et al., 2012). For a large set of problems, there is no analytical closed solution to this equation. Generally, a numerical procedure was applied to calculate the sound pressure field (Lins and Rochinha, 2009; Lan, 2005). The finite element (FE) simulation is a very powerful technique which can be applied to obtain an approximate solution to the Helmholtz equation. The deterministic simulation leads to an approximate and nominal solution of reality. In some cases, the behavior prediction of a system is especially difficult because of the variability induced by uncertainty. The challenge is to improve the performance of numerical simulations of guided acoustic propagation. Recently, the probabilistic modeling of mechanical problems has received attention of some researchers (Sepahvand and Marburg, 2014; Xia et al., 2015). All of them are looking for 
more robust models that take into account the random nature of some parameters: material properties, geometrical irregularities, boundary and initial conditions, operating conditions, loading. The numerical accuracy and error control have been employed in simulations for fluid structure interaction (Mansouri et al., 2013) vibroacoustic problems (Dammak et al., 20117b; Mansouri et al., 2012a,b; Sepahvand and Marburg, 2014), CFD research (Xiu and Karniadakis, 2003) and dynamic responses of engineering structures (Yang and Kessissoglou, 2013). The application of the FE method to the Helmholtz equation has also been an object of certain studies concerning the error estimation and propagation (Ihlenburg and babuska, 1995). In (Lins and Rochinha, 2009), the solution of acoustic problems was resolved numerically when the boundary conditions presented uncertainty. Nevertheless, according to (Lepage, 2006), there is a relative lack of information about how statistical distributions of some variables influence the distributional properties of the acoustic response. Based on literature reviews, one can distinguish two ways to include stochastical behavior in finite element simulations: the first is a statistical approach or a sampling method, like the Monte Carlo (MC) technique (Hurtado and Alvarez, 2012). In this method, a large number of samples of input variables are required for reasonable accuracy. The problem is then solved for each realization. This technique allows one to obtain the entire probability density function of any system variable. It is widely used since it is easier to implement and very robust. However, a huge number of realizations to be solved could lead to a prohibitive computational cost. The second probabilistic tool is a non-statistic approach or a non-sampling method, which results in analytical treatment of the stochastic process. It consists in the polynomial chaos (Fisherand Bhattacharya, 2008; Creamer, 2006; Ng and Eldred, 2012). It is a more efficient tool due to discretization of random parameters by a set of limited realizations. This theory was initiated by Ghanem and Spanos (1991) who used expansion in Wiener-Hermite polynomials to model stochastic processes with Gaussian random variables (Wiener, 1938). The convergence of such an expansion in the mean square sense has been shown (Cameron and Martin, 1947) and generalized to various continuous and discrete distributions using orthogonal polynomials following the so called Askey-scheme (Xiu and Karniadakis, 2002). This general extension is known as generalized polynomial chaos (gPC) (Wan and Karniadakis, 2006). Polynomial chaos gives a mathematical framework to separate the stochastic components of a system response from deterministic ones. According to (Xiu and Karniadakis, 2002, 2003), polynomial chaos did not receive much attention for a long time. In the numerical aspect, one can distinguish intrusive and non-intrusive methods. The first method is applied to systems in which the governing equations are known. In this case, the stochastic equations are used to generate a set of deterministic equations using the Galerkin method (Ghanem and Spanos, 1991), which are difficult to implement. Nonintrusive technique seems to be more efficient since it only requires simulations corresponding to particular samples of the random parameters, and no modifications are needed on the system model (Nechak et al., 2013). It worth mentioning that works on numerical simulation of sound propagation in a three-dimensional duct coupled with uncertainty analysis remains infrequent. In (Taktak et al., 2011), a numerical method of the modeling of sound propagation in circular and rectangular cross-section ducts in the presence of flow was developed and presented. The pressure acoustic field inside the duct was determined for several incident acoustic modes. In (Kesentini et al., 2015), the wave finite element method was applied to study guided acoustical propagation. In those studies, uncertainties on the fluid-structure interaction were neglected.

In this paper, we aim at increasing the performance prediction for acoustic propagation systems that will operate in the presence of the inevitable parameters uncertainty associated with the geometric and material properties. The stochastic methods discussed above, MC and $\mathrm{gPC}$, are implemented and integrated in finite element simulation for a circular cross-section duct. The simulation results are discussed, compared and validated with literature. 


\section{Governing equations of the physical problem}

\subsection{Acoustic propagation in a cylindrical duct}

In this Section, the governing equations of sound propagation in circular cross-section ducts are presented in Fig. 1. The equation governing the acoustic behavior of a fluid is the Helmholtz

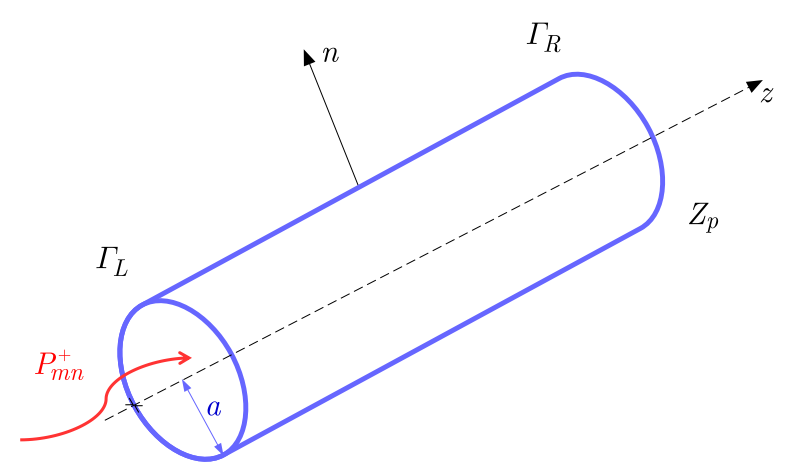

Fig. 1. Description of the cylindrical guide

equation (Taktak et al., 2011; Kim and Nelson, 2004)

$$
\Delta p_{i}+k^{2} p_{i}=0
$$

where $\Delta$ is the Laplacian operator, $p_{i}$ is the acoustic pressure inside the studied duct and $k$ is the total wave number. It is well noticed that the resolution of this equation depends on the duct geometry. In this work, a cylindrical cross-section duct is considered. For this kind of duct, the resolution Helmholtz equation in the cylindrical coordinates system is carried out by the variables separation method. The acoustic pressure is expressed as

$$
P\left(r_{i}, \theta, z\right)=R\left(r_{i}\right) \Theta(\theta) Z(z)
$$

where

$$
Z(z)=A \mathrm{e}^{\mathrm{j} k_{z} z}+B \mathrm{e}^{-\mathrm{j} k_{z} z} \quad \Theta(\theta)=\theta_{1} \mathrm{e}^{-\mathrm{j} m \theta}+\theta_{2} \mathrm{e}^{\mathrm{j} m \theta} \quad R\left(r_{i}\right)=C J_{m}\left(k_{r} r_{i}\right)
$$

where $A, B, \theta_{1,2}$ and $C$ are constants depending on boundaries conditions. The wave numbers are related by the dispersion relation (Taktak, 2008)

$$
\Gamma_{m n}^{2}+k_{m n}^{2}-k^{2}=0
$$

In the case of a rigid wall, the radial wave number is defined by

$$
\Gamma_{m n}=\frac{\chi_{m n}}{a}
$$

where $\chi_{m n}$ is the $n$-th root of the derivative of $J_{m}$ - the Bessel function of the first kind of the order $m$. Thus the modal analysis can be applied (Dammak et al., 2017a; Blazejewski, 2013; Blazejewski et al., 2014) and the acoustic pressure field inside the duct is obtained as

$$
P\left(r_{i}, \theta, z, t\right)=\sum_{m=0}^{\infty} \sum_{n=0}^{\infty} P_{m n}(z) \Psi_{m n}\left(r_{i}, \theta\right) \mathrm{e}^{-\mathrm{j} \omega t}
$$

where $P_{m n}$ are the modal coefficients associated modes $(m, n)$ defined by

$$
P_{m n}(z)=A_{m n} \mathrm{e}^{-\mathrm{j} k_{m n} z}+B_{m n} \mathrm{e}^{\mathrm{j} k_{m n} z}
$$

and $\Psi_{m n}$ are the eigenfunctions of the enclosure (Blazejewski et al., 2014; Meissner, 2008), which satisfy the Helmholtz equation and can be expressed as

$$
\Psi_{m n}\left(r_{i}, \theta\right)=J_{m}\left(\chi_{m n} \frac{r_{i}}{a}\right) \mathrm{e}^{\mathrm{j} m \theta}
$$

The Helmholtz equation will be solved numerically in Section 3. 


\subsection{Uncertainty modeling}

In this Section, we introduce two classical techniques for representing random processes: the generalized Polynomial Chaos (gPC) and the Monte Carlo (MC) technique.

\subsubsection{Polynomial chaos}

The Polynomial Chaos was originally developed by Wiener (1938). It is a stochastic method based on spectral representation of the uncertainty. The PC decomposes a random function (or variable) into separable deterministic and stochastic components. Here, a brief mathematical review of this approach will be given. For instance, considering any random variable $\lambda_{i}$ such as velocity, density, or pressure in a stochastic fluid dynamics problem, one can write (Nechak et al., 2013)

$$
\lambda_{i}(x, \xi)=\sum_{j=0}^{\infty} \bar{\lambda}_{i, j}(x) \phi_{j}(\xi)
$$

where $\xi$ is the random variable vector with a known joint density function $W(\xi), \lambda_{i, j}$ is the deterministic component and $\phi_{j}(\xi)$ is the orthogonal polynomial function satisfying the orthogonality relation

$$
\left\langle\phi_{i}, \phi_{j}\right\rangle=\int \phi_{i} \phi_{j} W(\xi) d \xi= \begin{cases}0 & \text { if } i \neq j \\ \left\langle\phi_{i}, \phi_{j}\right\rangle & \text { if } i=j\end{cases}
$$

where $\langle\cdot\rangle$ is the internal product operator. As a series expansion to infinity cannot be used in practice, the sum is truncated to a finite number of terms $N_{p}$, which is shown to be dependent on the gPC order $p$, and the stochastic dimension $r$ denoting the number of uncertain parameters

$$
\lambda_{i}(x, \xi)=\sum_{j=0}^{N_{p}} \bar{\lambda}_{i, j}(x) \phi_{j}(\xi)
$$

with

$$
N_{p}=\frac{(p+r) !}{p ! r !}-1
$$

For a random variable with certain distribution, the orthogonal function $\phi_{j}$ can be chosen in such a way that its weight function has the same form as the probability function $W(\xi)$. Then, computing $\lambda_{i}$ is transformed into the problem of finding the coefficients $\bar{\lambda}_{i, j}$ of its truncated expansion (Smith et al., 2007). To extend the application of the polynomial chaos theory to propagation of continuous non-normal input uncertainty distributions, Xiu and Karniadakis (2003) used a set of polynomials known as the Askey scheme to obtain the Wiener-Askey generalized Polynomial Chaos. Table 1 shows commonly used, Legendre, Hermite, and Laguerre polynomials and the associated probability density functions (PDF) included the Askey scheme. Legendre and Laguerre polynomials are optimal basis functions for uniform and exponential input uncertainty distributions respectively, whereas the Hermite polynomials are optimal for the normal distributions in terms of the convergence of the statistics.

The intrusive and non-intrusive approaches are generally defined to calculate these coefficients called stochastic modes. The non-intrusive approach seems to be more efficient since it only requires simulations corresponding to particular samples of random variables and it needs no modifications of the stochastic model, contrary to the intrusive approach. That is why only the non-intrusive approach is considered in this paper. This approach considers the deterministic model as a black-box and approximates the stochastic coefficients with formulas based 
Table 1. Correspondence between the type of distribution and the type of base of chaos

\begin{tabular}{|c|c|c|c|c|}
\hline Distribution & Density function & Polynomial & Weight function $W(\xi)$ & Support range \\
\hline \hline Uniform & $\frac{1}{2}$ & Legendre $L_{e}(\xi)$ & 1 & {$[-1,1]$} \\
\hline Normal & $\frac{1}{\sqrt{2 \pi}} \exp \left(\frac{-\xi^{2}}{2}\right)$ & Hermite $H_{n}(\xi)$ & $\exp \left(\frac{-\xi^{2}}{2}\right)$ & {$[-\infty,+\infty]$} \\
\hline Exponential & $\exp (-\xi)$ & Lagrange $L_{a}(\xi)$ & $\exp (-\xi)$ & {$[0,+\infty]$} \\
\hline
\end{tabular}

on deterministic code assessment. The spectral projection (NISP) and regression are the main non-intrusive polynomial chaos methods used for uncertainty quantification. In the technique NISP (Ng and Eldred, 2012), once the solution is expressed in the base of polynomial chaos following general expression (2.11), it is projected, which determines stochastic coefficients as

$$
\bar{\lambda}_{i, j}(x)=\frac{\left\langle\lambda_{i}(x, \xi), \phi_{j}(\xi)\right\rangle}{\left\langle\phi_{j}(\xi), \phi_{j}(\xi)\right\rangle}=\frac{1}{\left\langle\phi_{j}^{2}\right\rangle} \int \lambda_{i}(x, \xi) \phi_{j}(\xi) W(\xi) d \xi \quad j=0, \ldots, N_{p}
$$

The regression method (Blatman and Sudret, 2008) consists in calculating the stochastic coefficients so as to minimize the least squares sense, the gap $\varepsilon$ between the solution of the stochastic model and its approximation in the base of generalized polynomial chaos

$$
\varepsilon=\sum_{k=1}^{Q}\left[\lambda_{i}\left(x, \xi^{(k)}\right)-\sum_{j=0}^{N_{p}} \bar{\lambda}_{i, j}(x) \phi_{j}\left(\xi^{(k)}\right)\right]
$$

The $\xi^{(k)}$ may be selected from the roots of a polynomial with the condition $Q>N p+1$, with $Q$ being the number of Gauss points. By designating $\bar{\lambda}_{i, j}=\left(\bar{\lambda}_{i, 0}, \ldots, \bar{\lambda}_{i, N_{p}}\right)^{\mathrm{T}}$, the vector of modal coefficients, $\mathbf{Z}$ the matrix of elements $Z_{q, l}=\phi_{j}\left(\xi^{(q)}\right)$ and $\boldsymbol{\lambda}_{i}=\left(\lambda_{i}\left(x, \xi^{(1)}\right), \ldots, \lambda_{i}\left(x, \xi^{(q)}\right)\right)$ the vector corresponding to the game simulations $\boldsymbol{\xi}^{(q)}$. and if the matrix $\mathbf{Z}^{\mathrm{T}} \mathbf{Z}$ is non-singular then the optimal solution of the classical least squares problem is given by

$$
\overline{\boldsymbol{\lambda}}_{i, j}=\left(\mathbf{Z}^{\mathrm{T}} \mathbf{Z}\right)^{-1} \mathbf{Z}^{\mathrm{T}} \lambda_{i}
$$

The quality of the solution depends on the conditioning of the matrix $\left(\mathbf{Z}^{\mathrm{T}} \mathbf{Z}\right)$ called the Fischer matrix.

\subsubsection{Monte Carlo theory}

The MC method provides successive resolutions of a deterministic system incorporating uncertain parameters modeled by random variables. It generates, for all uncertain parameters and according to their probability distributions and their correlations, random simples. For each draw, a set of parameters is obtained and a deterministic calculation, following numerical (FES) or analytical models well defined, is made. The main advantage of this method is that it can be applied to any system, whatever is its size and complexity (linear, non-linear, etc.). A reasonable accuracy of the results requires a large number of draws which makes the MC method prohibitive in terms of computational cost. The standard MC approach considers functions of the following form

$$
\mathbf{Y}=M(\mathbf{X})
$$

where $M$ represents the model under consideration, $\mathbf{X}=\left[X_{1}, X_{2}, \ldots, X_{n}\right]^{\mathrm{T}}$ is a vector of uncertain input parameters and $\mathbf{Y}$ represents the vector of estimated outputs that will be a random vector. The algorithm of this method can be summarized in 5 steps: 
Step 1: probabilistic identification of uncertain parameters in the model;

Step 2: sampling and random generation of inputs following identified probabilistic distributions;

Step 3: spread of uncertainty i.e. of the data set resulting from step 2 into the model and determination of the corresponding outputs set;

Step 4: estimation of the outputs probabilistic distributions whose statistical characteristics are given by the mean value $\mu_{\gamma}$ and standard deviation $\sigma_{\gamma}$. These are calculated using a set of $N$ simulations as follows

$$
\mu_{\gamma}=\frac{1}{N} \sum_{i=1}^{N} M\left(X^{(j)}\right) \quad \sigma_{\gamma}^{2}=\frac{1}{N-1} \sum_{i=1}^{N}\left[M\left(X^{(j)}\right)-\mu_{\gamma}^{2}\right]
$$

Step 5: convergence analysis of the distribution of the model output.

\section{Numerical results}

Finite element simulations are carried out to study the acoustic propagation in a cylindrical duct. The acoustic pressure $P$ within a finite element can be written as

$$
P=\sum_{i=1}^{m} N_{i} P_{i}
$$

where $N_{i}$ is a set of linear shape functions, $P_{i}$ are acoustic nodal pressures at the node $i$, and $m$ is the number of nodes forming the element. For the pressure formulated acoustic elements, the finite element equation for the fluid in matrix form is

$$
\mathbf{M}_{f} \ddot{\mathbf{P}}+\mathbf{K}_{f} \mathbf{P}=\mathbf{F}_{f},
$$

where $\mathbf{M}_{f}$ is the equivalent fluid mass matrix, $\mathbf{K}_{f}$ is the equivalent fluid stiffness matrix, $\mathbf{F}_{f}$ is the vector of applied fluid loads, $\mathbf{P}$ is the vector of unknown nodal acoustic pressures, and $\ddot{\mathbf{P}}$ is the vector of the second derivative of acoustic pressure with respect to time.

Section 3.1 deals with numerical model validation without considering uncertainty. In Section 3.2 , the stochastic methods discussed above, $\mathrm{MC}$ and $\mathrm{gPC}$, are implemented and integrated in finite element simulation for the cylindrical duct. In this framework, geometrical (radius of the duct) and material properties (density) are presenting the probabilistic parameters.

\subsection{Deterministic model}

The chosen example consists in applying the modal pressure at the left boundary $\Gamma_{L}$ of a cylindrical duct, as indicated in Fig. 1. The geometric characteristics of the studied duct are: radius $a=0.02 \mathrm{~m}$ and length $L=0.25 \mathrm{~m}$. The magnitude of the imposed pressure is equal to $1 \mathrm{~Pa}$. At the end of the duct $\Gamma_{R}$, a normalized acoustic impedance is applied $\left(Z_{p}=2\right)$ to reflect the acoustic wave plane. The duct has been modelled using 8000FLUID30 elements. This is an entirely acoustic analysis and there are no active displacement degrees of freedom. In the following, the acoustic pressure fields obtained by the present numerical simulation without probabilistic approach is discussed. Only the real parts of the pressure are studied. The ANSYS Finite Element model of the duct is given in Fig. 2.

Figure 3 presents the pressure field inside the duct at several frequencies. The wave propagation is clear and the localization of the maximum of the real part of the acoustic pressure is varying as a function of the frequency. Frequency evolution of the pressure magnitude in one point inside the cylindrical duct is depicted in Fig. 4. The results greatly agree those shown in (Kesentini et al., 2015). 


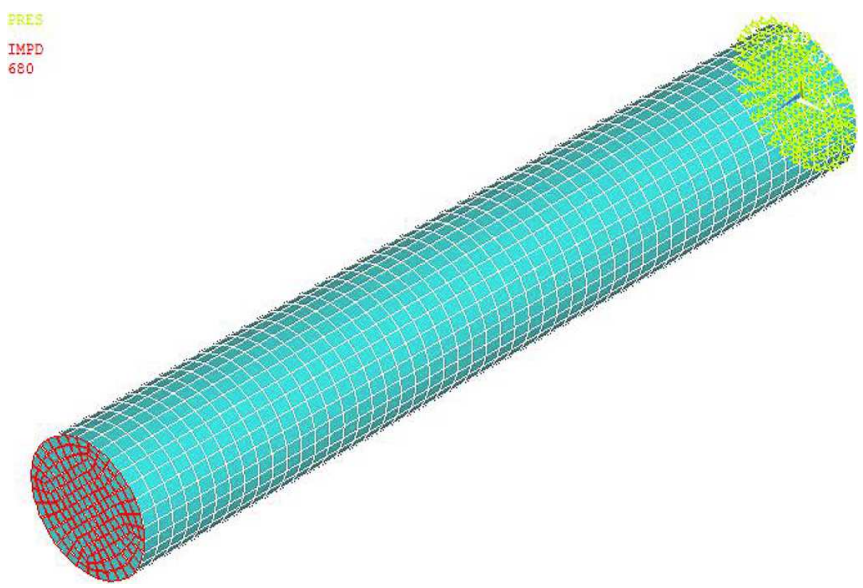

Fig. 2. ANSYS Finite Elements model of the cylindrical duct
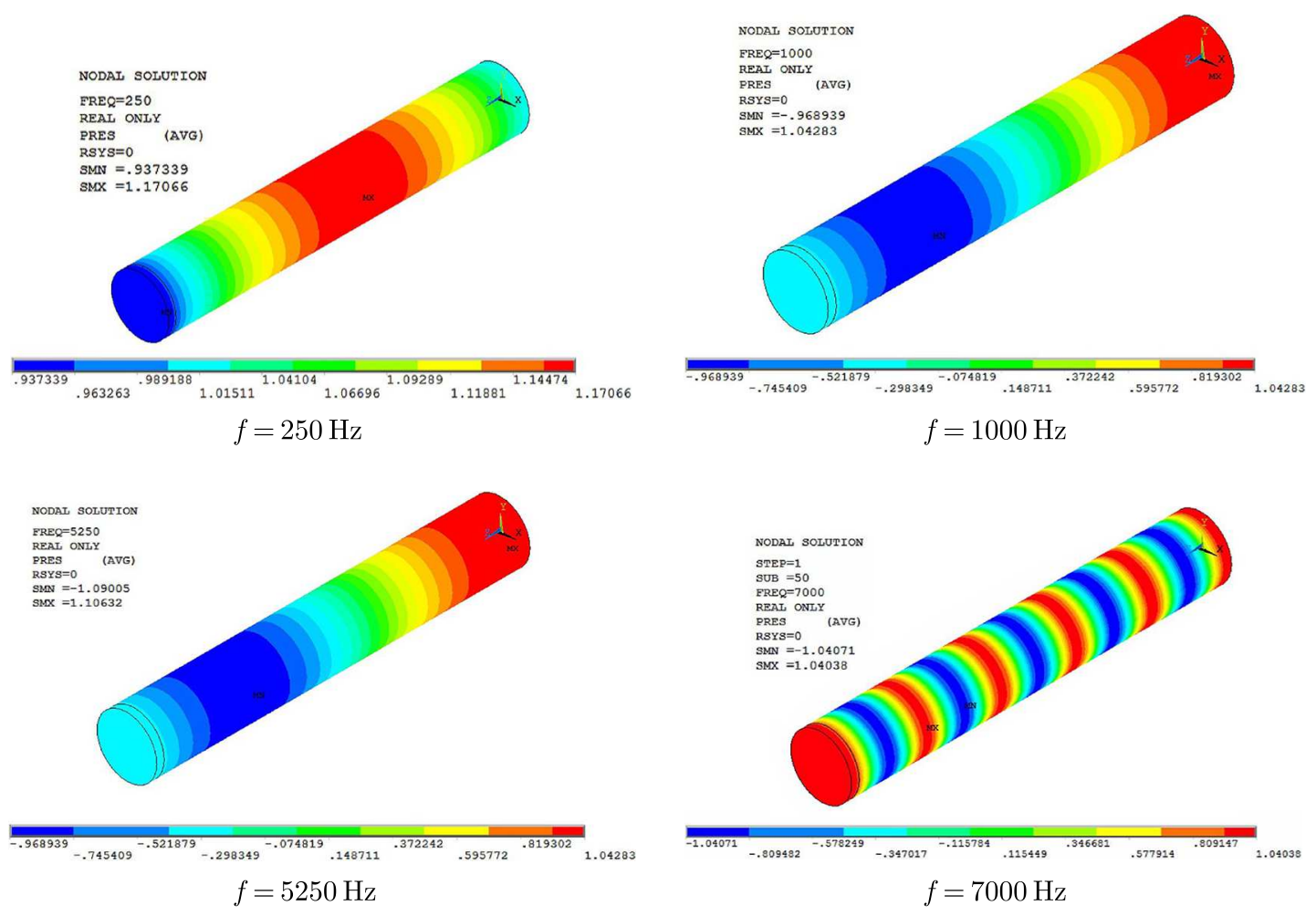

Fig. 3. Real part of the acoustic pressure inside of the studied cylindrical wall duct

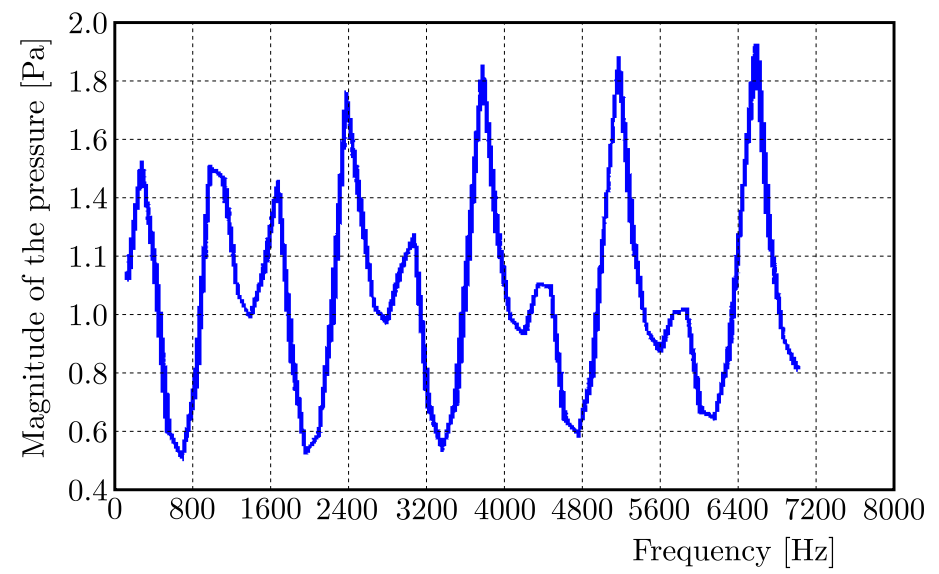

Fig. 4. Frequency evolution of the pressure magnitude in one point inside the cylindrical duct 


\subsection{Probabilistic analysis}

In this Section, the stochastic methods discussed above, MC and gPC, are implemented and integrated in finite element simulation, see Section 3.1. The objective is to enhance the performance prediction for acoustic propagation systems that will operate in the presence of the inevitable parameters uncertainty associated simultaneously with the geometric and material properties. The geometric uncertain parameter considered here is $a$, radius of the cylindrical duct. The physical uncertain parameter is density $\rho$. These parameters are chosen to be uniform random following a progressive nomination around their nominal values $\pm 5 \% ; a=\overline{a_{0}}+\xi \overline{a_{1}}$ and $\rho=\overline{\rho_{0}}+\xi \overline{\rho_{1}}$, where $\overline{a_{0}}, \overline{\rho_{0}}$ are the mean values, and $\overline{a_{1}}, \overline{\rho_{1}}$ are convenient constants. Using the Monte Carlo method to analyze the pressure field consists in creating a grid of numerical values from the probabilistic uncertain parameters and calculating the quantity of interest of the linearized system for each value of the grid. The case of uniform distribution of the uncertain parameters is considered. The quantity of interest is analyzed for 500 drawings. Figure 5 shows the distribution of the input variables $(a, \rho)$ in the case of uniform distribution of uncertainty.
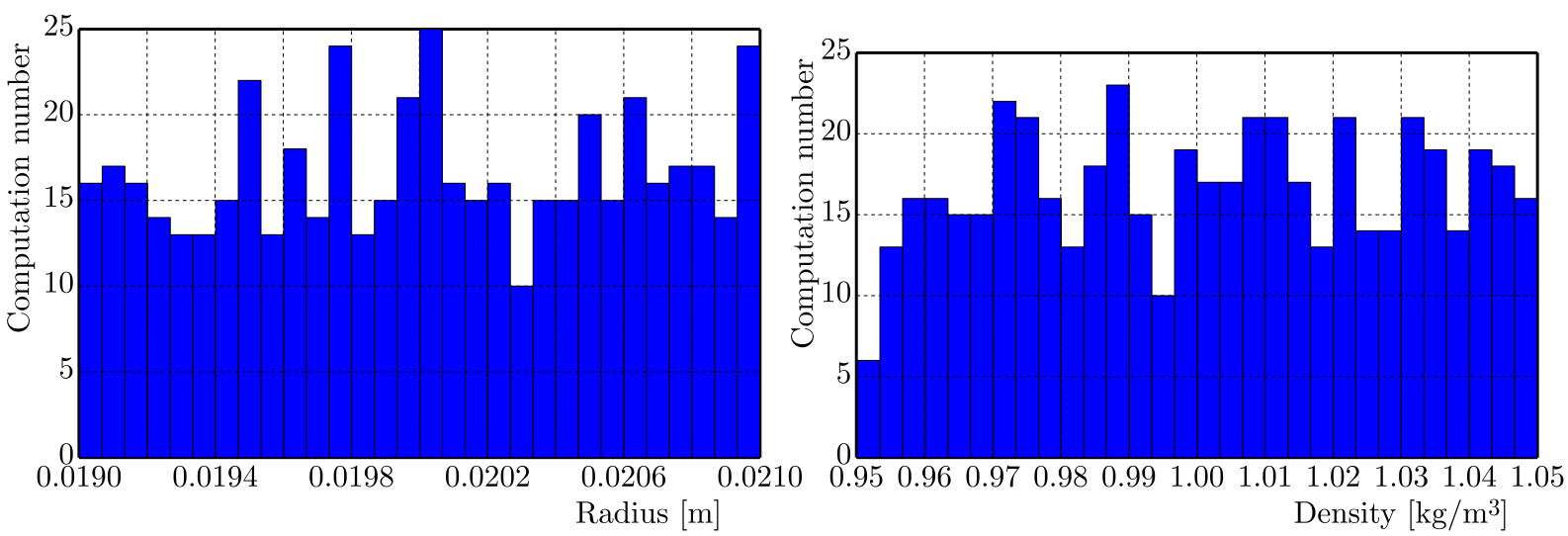

Fig. 5. Probability distribution of radius and density
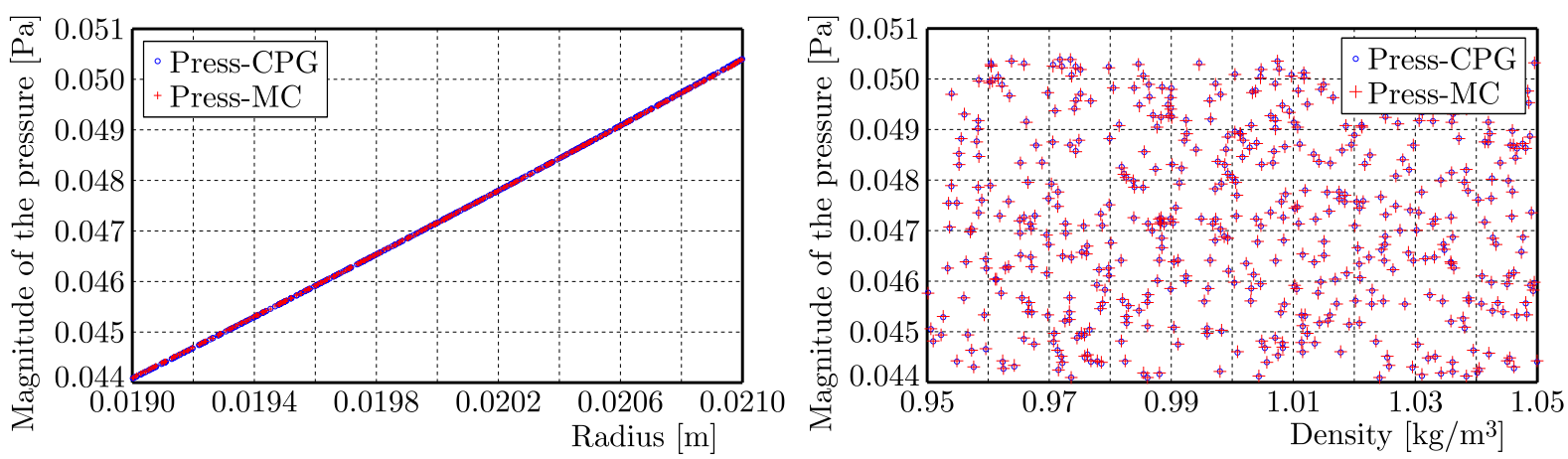

Fig. 6. Magnitude of the maximum pressure inside the duct as a function of geometric and material uncertain parameters

The results consist in the quantity of interest (the magnitude of pressure) plotted in Fig. 6 as a function of different duct radii and densities. The chaotic representation of the acoustic pressure is

$$
P\left(r_{i}, \theta, z, \xi\right)=\sum_{j=0}^{N_{p}} \bar{P}_{j}\left(r_{i}, \theta, z\right) \phi_{j}(\xi)
$$

The Monte Carlo representation for the acoustic pressure can be expressed as

$$
P_{M C}\left(r_{i}, \theta, z, \xi\right)=M(\mathbf{X})
$$


where $M$ is the finite element model and $\mathbf{X}=[a, \rho]^{\mathrm{T}}$ is the vector of input uncertain parameters. The probability distribution and the relative errors are shown in Figs. 7 and 8. All results are compared with the direct method of MC. Models based on gPC are constructed using $r$ uncertain parameters $(r=1 \rightarrow 2)$. It is well depicted from Fig. 6 that for $\pm 5 \%$ of radius variation $a$, the maximum pressure magnitude is varying linearly, justifying the choice of the drawing number in MC. In the case of variability within density, one can remark that radius has a more effect on the pressure magnitude than density.

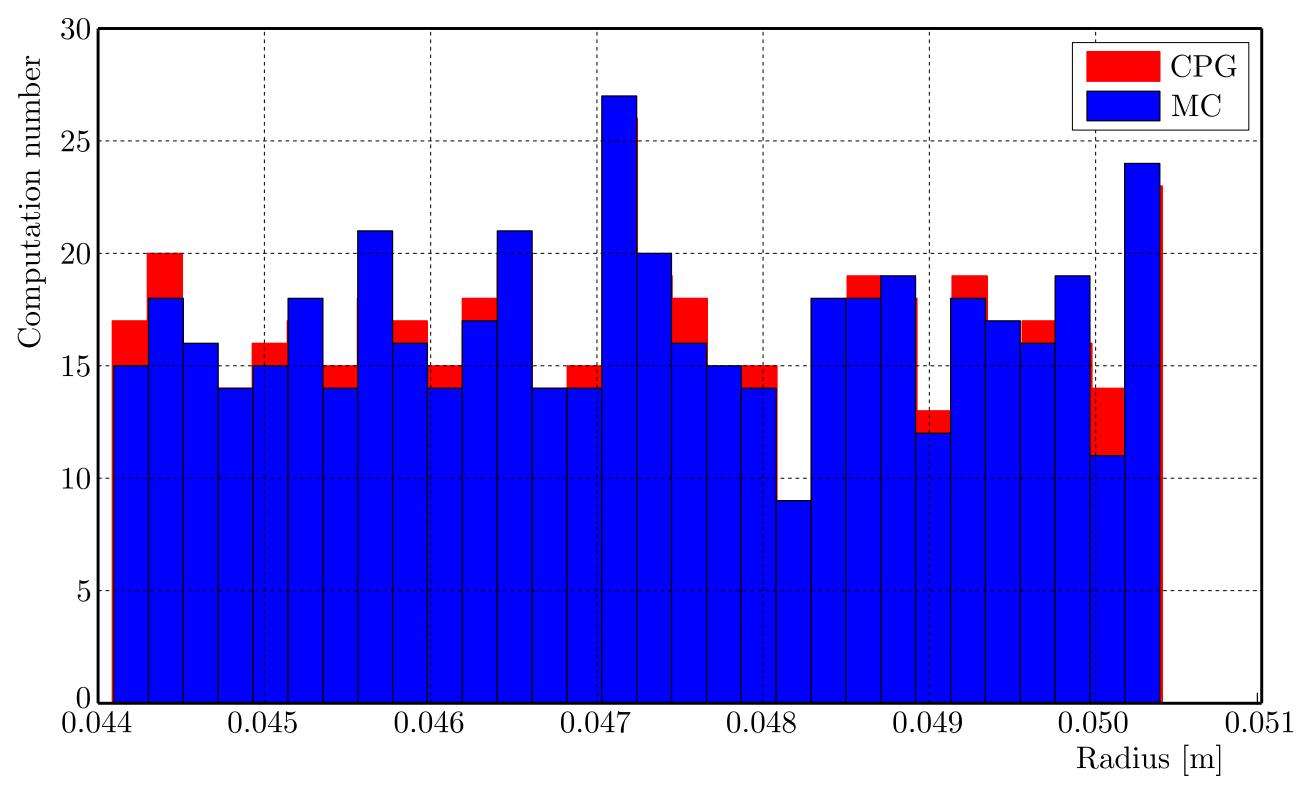

Fig. 7. Probability distribution of pressure

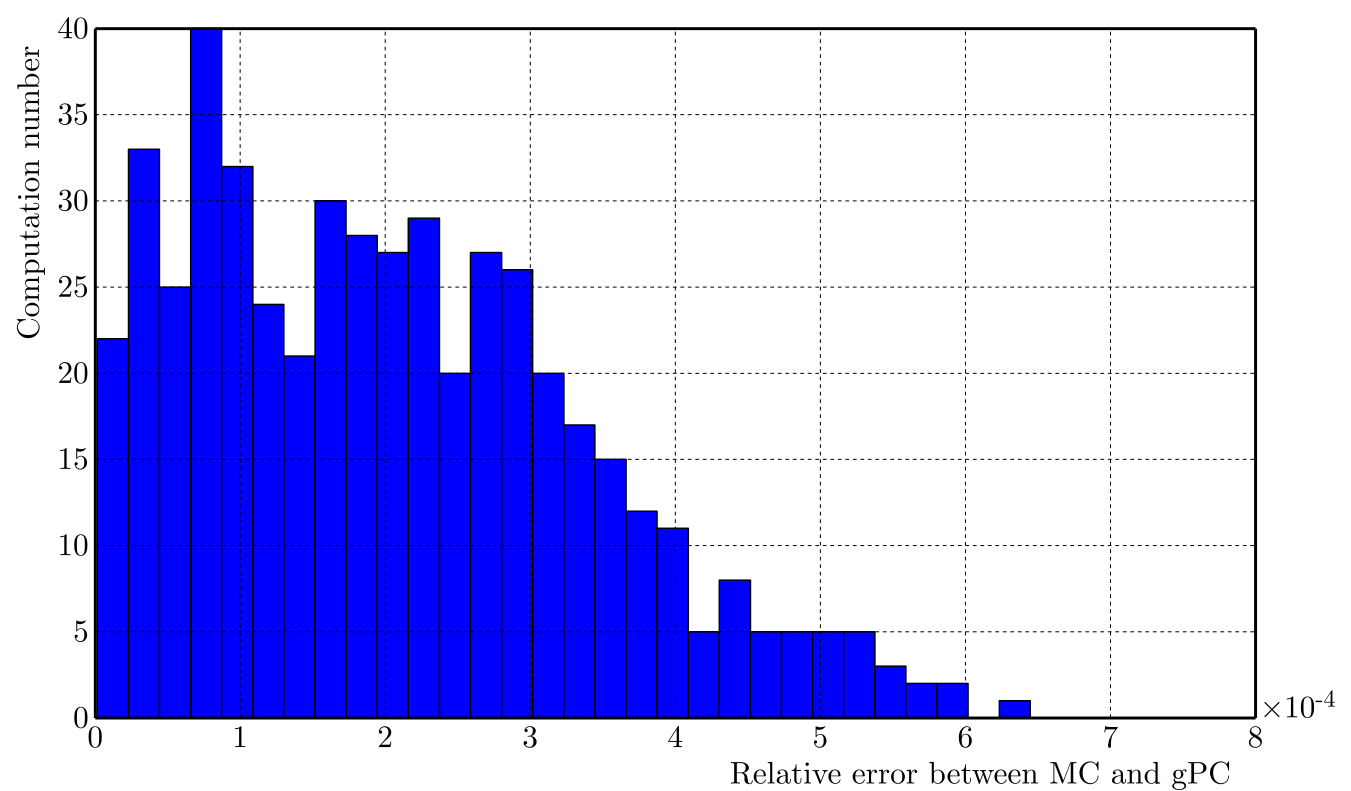

Fig. 8. Histogram of the relative error of pressure

We plot in Fig. 9 the mean value of the pressure as a function of the order $p$, for an uncertain parameter with variation of $\pm 5 \%$. It is noted that there is a convergence of the average from $p=4$. On can notice that the pressure distribution of the mean is very similar to that obtained when we use a deterministic model (Section 3.1). It is also proved that the results strongly depend on the frequency used. 


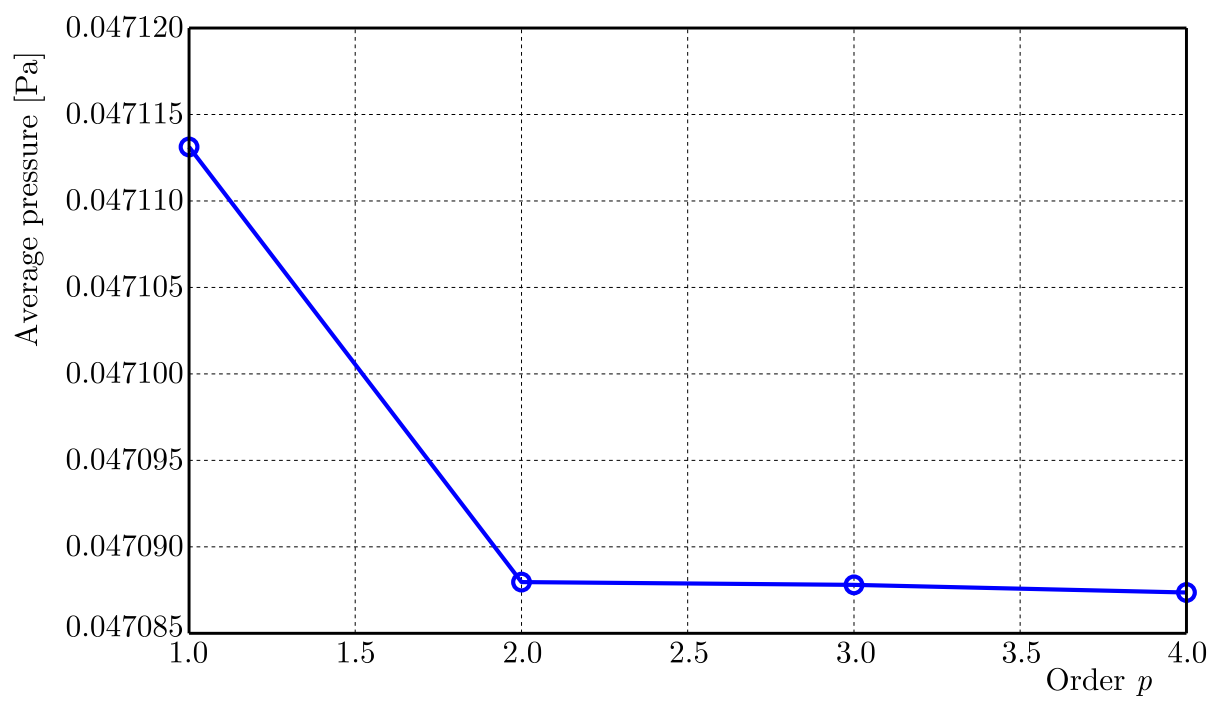

Fig. 9. Average of the pressure

Table 2 shows the main features of each technique. It is worth mentioning that the Monte Carlo technique is a very powerful method to solve complex systems with random parameters. In this work, 500 of samplings of two input variables are calculated and then the problem is solved for each sample of input variables. Nevertheless, according to results in Table 2, this technique has poor convergence for mean and standard deviation of the solution, requiring a large number of samples to achieve good precision in results, resulting in costly computation. This result is in a good agreement with (Nechak et al., 2013).

Table 2. Comparison between $\mathrm{MC}$ and $\mathrm{gPC}$

\begin{tabular}{|l|c|c|}
\hline \multicolumn{1}{|c|}{ Frequency $f=1000 \mathrm{~Hz}$} & Monte Carlo & $\mathrm{gPC}(r=2)$ \\
\hline \hline Order $p$ & - & 4 \\
\hline Number of simulations & 500 & 25 \\
\hline Maximum relative error between gPC and MC & - & $6.4 \mathrm{e}^{-4}$ \\
\hline Mean of the pressure $(\mathrm{Pa})$ & 0.047262 & 0.047262 \\
\hline Standard deviation & 0.001821 & 0.001826 \\
\hline Time $[\mathrm{s}]$ & 43438.96 & 0.5182 \\
\hline
\end{tabular}

For high frequencies that are greater than $5000 \mathrm{~Hz}$, Fig. 10 shows that the acoustic pressure is varying nonlinearly according to radius of the cylindrical duct. We plot in Figs. 11a and 11b, respectively, the mean value and the standard deviation of pressure as a function of the order $p$. It is clear that there is a convergence of these statistical results from $p=7$.

\section{Conclusions}

In this work, the MC method and the gPC have been coupled to FE simulation discussed above in order to calculate statistical data from output pressure field. In this paper, a numerical solution of the Helmholtz equation is proposed based on finite element simulation. This solution is coupled to probabilistic approaches, when physical parameters present uncertainty. The case of a cylindrical duct has been considered. The influence of uncertain variables on the pressure field has been discussed. The results using the polynomial chaos expansion method have been compared with the Monte Carlo technique. Convergence has been verified with comparisons against exact solutions and solutions from Monte Carlo simulations. As regards efficiency, gPC 


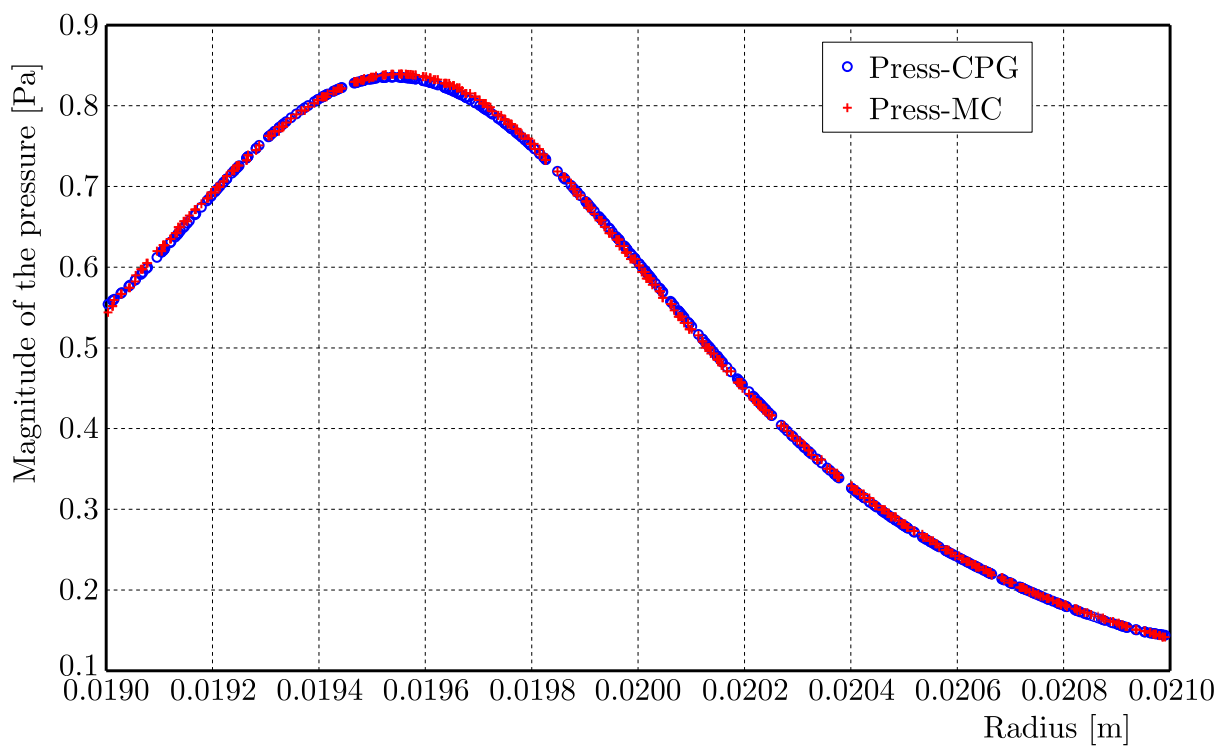

Fig. 10. Magnitude of the maximum pressure inside the duct as a function of radius at a high frequency

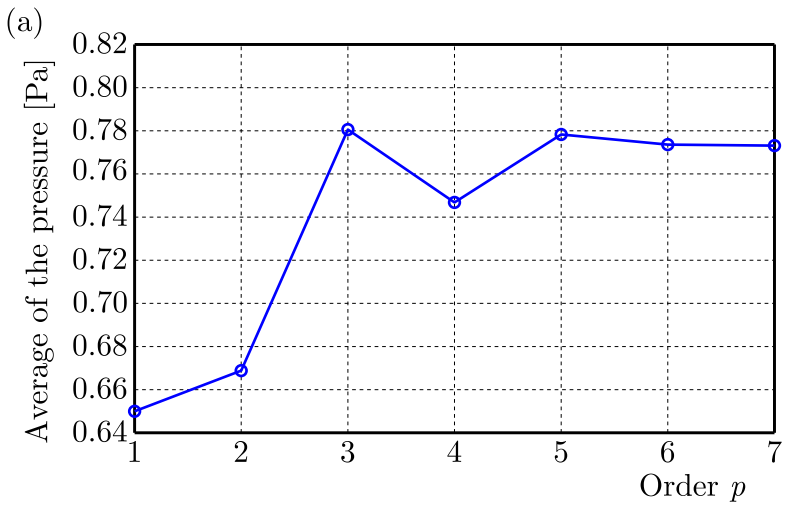

(b)

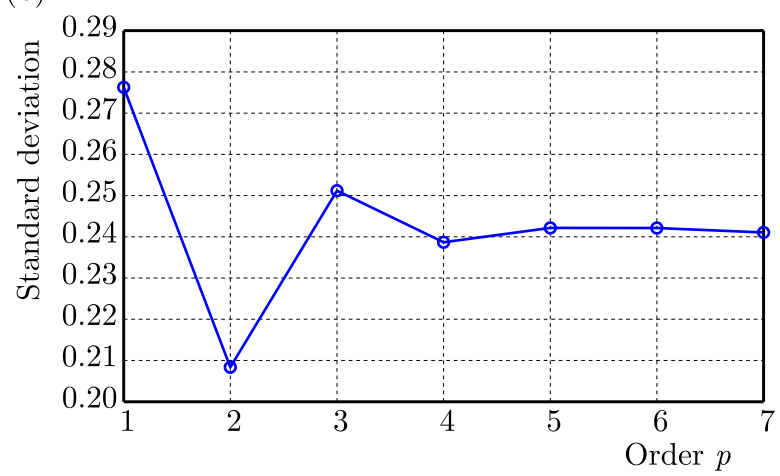

Fig. 11. (a) Average and (b) standard deviation of pressure

based simulation is computationally less expensive than the MC technique to generate the solution statistics. In the problems we have studied here, we can only make direct comparisons when using two random physical parameters, and no interaction is considered. The future track of work will consist in the study of uncertainty of the fluid-structure interaction when several uncertain input variables are included.

\section{References}

1. Blatman G., Sudret B., 2008, Sparse polynomial chaos expansions and adaptive stochastic finite elements using a regression approach, C.R. Mecanique, 336, 518-523

2. Blazejewski A., 2013, Modal analysis application in passive sound level control inside bounded space in steady state, 12th Conference of Dynamical Systems Theory and Applications, Dynamical Systems-Application, 409-420

3. Blazejewski A., Koziol P., Luczak M., 2014, Acoustical analysis of enclosure as initial approach to vehicle induced noise analysis comparatevely using STFT and wavelets, Archives of Acoustics, 39, 385-394

4. Cameron H., Martin W., 1947, The orthogonal development of non linear functionals in series of Fourier-Hermite functional, Annals of Mathematics, 48, 385-392 
5. Cheung Y.F., Jin W.G., 1991, Solution of Helmholtz equation by Trefftz method, International Journal for Numerical Methods in Engineering, 32, 63-78

6. Creamer D.B., 2006, On using polynomial chaos for modeling uncertainty in acoustic propagation, Journal of the Acoustical Society of America, 119, 4, 1979-1994

7. Dammak K., Elhami A., Koubaa S., Walha L., Haddar M., 2017a, Reliability based design optimization of coupled acoustic-structure system using generalized polynomial chaos, International Journal of Mechanical Sciences, 134, 75-84

8. Dammak K., Koubaa S., Elhami A., Walha L., Haddar M., 2017b, Numerical modelling of vibro-acoustic problem in presence of uncertainty: Application to a vehicle cabin, Applied Acoustics, DOI: $10.1016 /$ j.apacoust.2017.06.001

9. Fisher J., Bhattacharya R., 2008, Stability analysis of stochastic systems using polynomial chaos, American Control Conference

10. Ghanem R.G., Spanos P.D., 1991, Stochastic Finite Elements: a Spectral Approach, Dover Publication INC

11. Hurtado J.E., Alvarez D.A., 2012, The encounter of interval and probabilistic approaches to structural reliability at the design point, Computer Methods in Applied Mechanics and Engineering, 225-228, 74-94

12. Ihlenburg F., BabuskA I., 1995, Finite element solution of the Helmholtz equation with high wave number. Part I: The h-version of the FEM, Computers and Mathematics with Applications, 30, 9-37

13. Kesentini A., Taktak M., Ben Souf M.A., Bareille O., Ichchou M.N., Haddar M., 2015, Computation of the scattering matrix of guided acoustical propagation by the Wave Finite Element approach, Applied Acoustics

14. Kim Y., Nelson P.A., 2004, Estimation of acoustic source strengt hwith in a cylindrical duct by inverse methods, Journal of Sound and Vibration, 275, 391-413

15. LAN J.H., 2005, Validation of 3D acoustic propagation code with analytical and experimental results, 11th AIAA/CEAS Aeroacoustics Conference

16. Lepage K.D., 2006, Estimation of acoustic propagation uncertainty through polynomial chaos expansions, 9th International Conference on Information Fusion

17. Lins E.F., Rochinha F.A., 2009, Analysis of uncertainty propagation in acoustics through a sparse grid collocation scheme, 20th International Congress of Mechanical Engineering

18. Mansouri M., Radi B., El-Hami A., 2012a, Reliability-based design optimization for the analysis of vibro-acoustic problems, Proceedings of the Eleventh International Conference on Computational Structures Technology

19. Mansouri M., Radi B., El-Hami A., 2013, Coupling modal synthesis and reliability for studying aero-acoustic problems, 21me Congrés Français de Mécanique

20. Mansouri M., Radi B., El-Hami A., Borza D., 2012b, Reliability analysis of vibro-acoustic problems, Proceedings of the 1st International Symposium on Uncertainty Quantification and Stochastic Modeling

21. Meissner M., 2008, Influence of wall absorption on low-frequency dependence of reverberation time in room of irregular shape, Applied Acoustics, 69, 583-590

22. Nark D.M., Farassat F., Pope D.S., Vatsa V., 2003, The Development of the Ducted Fan Noise Propagation and Radiation Code CDUCT-LARC, American Institute of Aeronautics and Astronautics

23. Nark D.M., Watson W.R., Jones M.G., 2005, An Investigation of Two Acoustic Propagation Codes for Three-Dimensional Geometries, American Institute of Aeronautics and Astronautics 
24. Nechak L., Berger S., Aubry E., 2013, Robust analysis of uncertain dynamic systems: combination of the centre manifold and polynomial chaos theories, WSEAS Transactions on Systems, 98, 386-395

25. NG L.W.T., Eldred M.S., 2012, Multifidelity uncertainty quantification using non-intrusive polynomial chaos and stochastic collocation, Structural Dynamics and Materials Conference

26. Sepahvand K., Marburg S., 2014, On uncertainty quantification in vibroacoustic problems, Proceedings of the 9th International Conference on Structural Dynamics, EURODYN

27. Smith A.H.C., Monti A., Member S., Ponci F., 2007, Indirect measurements via a polynomial chaos observer, IEEE Transactions on Instrumentation and Measurement, 56, 743-752

28. TAKTAK M., 2008, Mesure de la matrice de diffusion d'un tronçon traité cylindrique e recouvert par un materiaux: apllication á la mesure de son efficacité et à la détermination de son impédance homogéneisée, L'Université de Technologie de Compiègne

29. Taktak M., Jrad H., Karra C., Bentahar M., Haddar M., 2011, Numerical modeling of the acoustic propagation in a three-dimensional wave guide in the presence of flow, ACTA Acoustica United with Acoustica, 97, 354-465

30. Taktak M., Majdoub M.A., Bentahar M., Haddar M., 2012, Numerical modelling of the acoustic pressure inside an axisymmetric lined flow duct, Archives of Acoustics, 37, 151-160

31. Wan X., Karniadakis G.E., 2006, Multi-element generalized polynomial chaos for arbitrary probability measures, Journal of Scientific Computing, 28, 901-928

32. Wiener N., 1938, The homogeneous chaos, American Journal of Mathematics, 60, 897-936

33. XiA B., Yin S., Yu D., 2015, A new random interval method for response analysis of structural acoustic system with interval random variables, Applied Acoustics, 99, 31-42

34. Xiu D., Karniadakis G.E., 2002, The Wiener-Askey polynomial chaos for stochastic differential equations, Journal of Scientific Computing, 24, 619-644

35. Xiu D., Karniadakis G.E., 2003, Modeling uncertainty in flow simulations via generalized polynomial chaos, Journal of Computational Physics, 187, 137-167

36. Yang J., Kessissoglou N., 2013, Modal analysis of structures with uncertainties using polynomial chaos expansion, Proceedings of Acoustics 